\title{
Influence of nanostructured ITO films on surface recombination processes in silicon solar cells
}

\author{
V.P. Kostylyov, A.V. Sachenko, O.A. Serba, T.V. Slusar, V.M. Vlasyuk, \\ P.O. Tytarenko, V.V. Chernenko \\ V. Lashkaryov Institute of Semiconductor Physics, NAS of Ukraine, \\ 41, prospect Nauky, 03028 Kyiv, Ukraine; \\ Phone/fax: +38(044) 525 5788. E-mail: vkost@isp.kiev.ua
}

\begin{abstract}
This paper describes the results of comparative studies of illumination currentvoltage characteristics and spectral characteristics of silicon solar cells with rear location of the collector p-n-junction for the cases of non-passivated and passivated front illuminated surface. Passivation was performed by silicon dioxide layer or ITO layer. It was found that ITO layer surface passivation with formation of ITO/silicon heterojunction, unlike silicon dioxide layer passivation, leads to a significant reduction of the effective surface recombination velocity. It significantly increases the value of the internal quantum efficiency in the wavelength range from 550 to $1050 \mathrm{~nm}$ and, as a result, significantly increases the value of short-circuit current of solar cells.
\end{abstract}

Keywords: solar cell, internal quantum efficiency, external quantum efficiency, velocity of surface recombination, spectral dependence, isotype heterojunction.

Manuscript received 23.04.15; revised version received 14.08.15; accepted for publication 28.10.15; published online 03.12.15.

\section{Introduction}

Films of transparent conductive oxides (TCO) characterized by high optical transparence and electrical conductivity are now widely used in manufacturing upto-date optoelectronic devices, where they are applied for creation of transparent electrodes and antireflection layers. When using these films in silicon solar cells (SC), it is also important to solve the task of passivation of silicon surface with the aim to reduce the velocity of surface recombination and, as a result, to enhance the efficiency of these elements. In particular, firms Sanyo/Panasonic already use TCO films in industrial production of silicon SC based on the junction $a$ $\mathrm{Si}: \mathrm{H} / \mathrm{Si}$ (crystalline), which reach the efficiency up to $25.6 \%$ [1]. One of the diverse TCO materials is indium oxide doped with tin ITO that, starting from 1954, when ITO film with suitable combination of optical transparence and electrical conductivity was prepared
[2], is actively investigated with the aim of large-scale usage $[3,4]$.

On the other hand, it is wellknown that, to decrease the negative influence of surface recombination on performances of silicon SC, beside the method for passivation of silicon surface, widely used is the method for creation of near-surface junctions. While passivation of the silicon surface, which is realized by deposition of dielectric layers, decreases the velocity of surface recombination directly at the interface silicon-dielectric due to lowering the concentration of surface recombination centers, availability of of near-surface junctions provides a potential barrier that limits the influx of definite type charge carriers to surface recombination centers [5]. These near-surface junctions can be created due to formation of both isotype $n^{+}-n$ - or $p^{+}-p$-junctions and floating $p^{+}-n$ - or $n^{+}-p$-junctions $[6,7]$ near the surface. In the case of available near-surface junctions, researches operate with the notion of 
"effective surface recombination velocity" related to the boundary between the range of space charge and quasineutral bulk. At the same time, this decrease in the negative influence of surface recombination on silicon SC performances is studied insufficiently.

Therefore, this work was aimed at performing investigations of the efficiency of applied ITO layers for creation of the junction ITO/Si with the purpose to decrease both recombination (effective surface recombination velocity) and optical (coefficient of light reflection) losses in silicon $\mathrm{SC}$.

\section{Experimental methods}

Experimental investigations were performed using the SC samples with rear collector junction having the thiockness $400 \mu \mathrm{m}$. The samples were made from zone melting $n$-type silicon КБЕ-2 with the specific resistance close to $2 \mathrm{Ohm} \cdot \mathrm{cm}$. These samples had the frontal surface free of electrodes. It was provided by placing the aluminum contact metalization of the interdigital type on the rear surface of SC in the form of oppositely directed combs, when one of them provides contact to the base $n$ range of $\mathrm{SC}$, while the other - to the $p^{+}$-range created due to boron diffusion near the rear surface. Near the frontal surface, these SC had shallow isotype $n^{+}-n$ junction formed using phosphorus diffusion at the doping level approximately $10^{19} \mathrm{~cm}^{-3}$ in the $n^{+}$-layer. Besides, to provide surface passivation and lowering optical losses, the frontal surface of SC was covered with the layer of silicon dioxide of 110-nm thickness. The schematic view of the silicon SC with the rear placement of the collecting $p$ - $n$-junction and antirecombination $n^{+}-n$-junction is shown in Fig. 1.

The abovementioned SC samples served as the objects for investigations of light current-voltage characteristics (CVC), and the latter enabled to

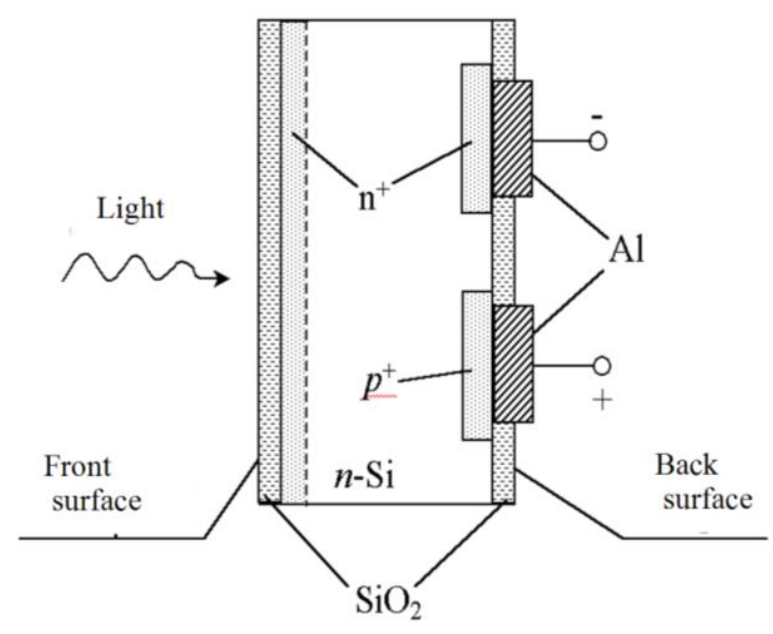

Fig. 1. Schematic representation of back junction, rear contact experimental silicon SC. determine the main photo-energetic characteristics of SC. Also, they allowed measuring the spectral dependences of the short-circuit current Isc through SC within the wavelength range $\Delta \lambda=400 \ldots 1200 \mathrm{~nm}$. These dependences were measured in the mode of automatic tuning the level of irradiance, which enabled to obtain the spectra of internal and external efficiencies. To determine the influence of the ITO/Si heterojunction formed on the front surface of SC on generationrecombination processes in it, the above characteristics were studied step-by-step, namely: first, for initial SC, then after removing the silicon dioxide layer from the front surface (by using the treatment in the concentrated fluorine acid) and, finally, after formation of a heterojunction at the surface of silicon SC by deposition of the ITO film with the thickness $75 \mathrm{~nm}$, applying the method of reactive ion sputtering the indium-tin target in argon-oxygen ambient. When processing in concentrated fluorine acid, SC were reliably protected, etchant was active only on the frontal surface. After processing, the samples were thoroughly washed in deionized water.

Such a choice of the experimental samples with the rear placement of barriers and contact metallization was related with their high sensitivity to surface and bulk recombination, changes of which we should register.

Light CVC, in particular, allow studying behavior of the short-circuit current, the value of which is defined by the efficiency of collection inherent to photogenerated charge carriers, and using the spectral dependences of the short-circuit current one can determine spectra of internal and external quantum efficiencies. The latter enables one to ascertain features of recombination processes at the near-surface range and in bulk, which, in turn, make an effect on the efficiency of collection of non-equilibrium charge carriers in SC.

Our measurements of light CVC and spectral dependences were performed using the equipment for photo-technical testing SC as well as installation for determination of relative spectral characteristics of photoconverters in the Photovoltaic Converters and Modules Test Center at V.Ye. Lashkaryov Institute of Semiconductor Physics, NAS of Ukraine.

The refraction index and thickness of ITO films were determined using a laser ellipsometer at the wavelength $632.8 \mathrm{~nm}$.

\section{Results and discussion}

Shown in Fig. 2 are typical light CVC of the studied SC obtained under the spectral conditions AM1.5 (irradiance $P_{L}=1000 \mathrm{~W} / \mathrm{m}^{2}$, temperature $T=25^{\circ} \mathrm{C}$ ) starting from the initial one to the following after removing the silicon dioxide layer and after depositing the ITO film. The values of SC photoelectric parameters determined using these light CVC are adduced in Table 1. And Fig. 3 shows the spectral dependences of internal quantum efficiency of the same SC after identical treatment stages. 


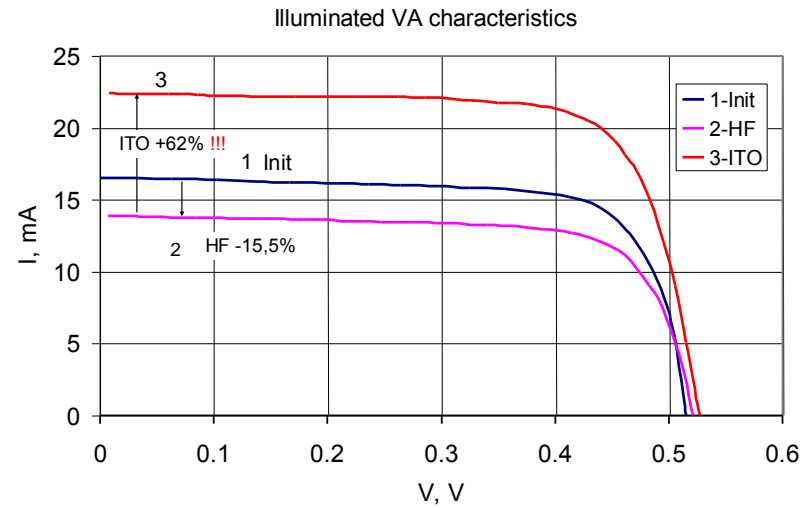

Fig. 2. Light current-voltage characteristics of $\mathrm{SC}$ at different stages of research: initial $\mathrm{SC}(1)$, after removing the $\mathrm{SiO} 2$ layer (2) and after formation of the ITO/Si heterojunction.

Table 1.

\begin{tabular}{|l|l|l|l|}
\hline $\begin{array}{l}\text { Light CVC } \\
\text { (Fig. 2) }\end{array}$ & $\begin{array}{l}\text { Short- } \\
\text { circuit } \\
\text { current, } \\
I_{s c}, \mathrm{~mA}\end{array}$ & $\begin{array}{l}\text { Open- } \\
\text { circuit } \\
\text { voltage, } \\
V_{o c}, \mathrm{~V}\end{array}$ & $\begin{array}{l}\text { Fill } \\
\text { factor } \\
\text { of light } \\
\text { CVC, } K_{f}\end{array}$ \\
\hline Initial & 16.43 & 0.515 & 0.75 \\
\hline $\begin{array}{l}\text { After removing } \\
\text { the } \mathrm{SiO}_{2} \text { layer }\end{array}$ & 13.89 & 0.521 & 0.75 \\
\hline $\begin{array}{l}\text { After deposition } \\
\text { of the ITO film }\end{array}$ & 22.43 & 0.527 & 0.75 \\
\hline
\end{tabular}

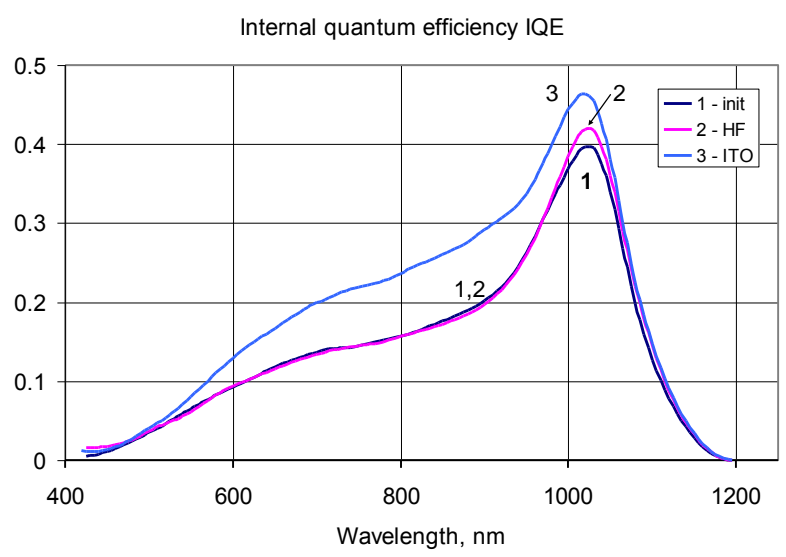

Fig. 3. Spectral dependencies of internal quantum efficiencies - IQE of SC obtained at different stages of researches: initial SC (1), after removing the $\mathrm{SiO} 2$ layer (2) and after formation of the ITO/Si heterojunction.

Our ellipsometric investigations have shown that ITO films are characterized by the values of refraction index lying within the range $n=1.95 \ldots 2.0$, while the thickness is close to $75 \mathrm{~nm}$. This thickness was chosen with account of optimal reduction of light reflection from silicon surface under conditions AM 1.5.

As seen from Fig. 2 and Table 1, removing the $\mathrm{SiO}_{2}$ layer from the frontal surface of $\mathrm{SC}$ results in decreasing the short-circuit current $I_{s c}$ by the value approximately $15.5 \%$, while deposition of the ITO film - to considerable growth of this parameter. The shortcircuit current value in the sample with ITO film exceeds that for SC with the removed silicon dioxide layer by approximately $62 \%$ and is considerably higher than the value for the initial $\mathrm{SC}$ sample having the $\mathrm{SiO}_{2}$ layer on its frontal surface. In this case, the open-circuit voltage is monotonically increased from $515 \mathrm{mV}$ in the initial sample up to $527 \mathrm{mV}$ after deposition of the ITO layer. It should be noted that for all the stages of experiment the fill factor FF of light CVC was kept unchanged and remained the same as that for the initial light CVC, namely 0.75 .

Spectral dependences of the internal quantum efficiency (IQE) at various stages of investigations changed as follows from Fig. 3. At the very beginning, after removing the silicon dioxide layer from the SC front surface, there takes place a weak increase of the internal quantum efficiency only near the maximum of dependence, and then, after deposition of the ITO layer on this surface, the IQE spectral characteristics is essentially changed as compared with previous dependences. It is pronounced in the considerable increase of the internal quantum efficiency within the wavelength range $550 \ldots 1050 \mathrm{~nm}$ and near the maximum of characteristic, in particular. It should be noted that light reflection has no influence on IQE values, since in the case of non-absorbing layers

$I Q E=\frac{E Q E}{1-R}$,

where EQE is the external quantum efficiency, and $R-$ reflection coefficient of the SC surface.

Similar results were observed in all the studied SC.

It is known that the value of surface recombination velocity for the non-passivated silicon surface considerably exceeds the respective value for the system $\mathrm{Si}-\mathrm{SiO}_{2}$. But the found changes in the short-circuit current values after removing the $\mathrm{SiO}_{2}$ layer are indicative of the fact that these changes are caused just by varying optical characteristics of the SC front surface (i.e., removal of the antireflection coating), while any change in the value of effective velocity of surface recombination $S_{\text {eff }}$, valid for the boundary between the space charge region and quasi-neutral bulk, is not observed. It is confirmed by practical absence of changes in respective spectral dependences for the internal quantum efficiency. We believe that it is caused by availability of the isotype $n^{+}-n$-junction at the SC front surface.

The found in this work approximately $62 \%$ increase in the value of $I_{\mathrm{sc}}$ current after deposition of ITO layer on the SC frontal surface and formation of the heterojunction ITO/silicon cannot be explained only by improvement of optical characteristics inherent to the SC frontal surface. It means that there takes place a considerable decrease in the value of effective surface recombination velocity $S_{\text {eff. }}$ It is unambiguously confirmed by the considerable increase in the internal quantum efficiency within the wavelength range 
$550 \ldots 1050 \mathrm{~nm}$, which is clearly seen from juxtaposition of the spectral dependences obtained for the SC samples before and after deposition of ITO layer (Fig. 3). Treatment of the experimental dependences $\operatorname{IQE}(\lambda)$ by using the method [9] has shown that, after formation of the heterojunction ITO/silicon, there takes place approximately 3-fold decrease in the value of effective surface recombination velocity. More exact ascertaining all the mechanisms responsible for the decrease of this value $S_{\text {eff }}$ after deposition of ITO layer requires additional theoretical investigations that are now performed.

\section{Conclusions}

It has been founded experimentally that effective surface recombination velocity Seff both on the passivated by thermal $\mathrm{SiO} 2$ film front surface with isotype $n+-n$ junction of the back junction back contacts SC, and after its removal remains approximately constant. At the same time, formation of the ITO/silicon heterojunction on this surface results in considerable decrease of Seff and, consequently, in essential growth of the internal quantum efficiency value within the wavelength range $550 \ldots 1050 \mathrm{~nm}$.

\section{References}

1. K. Masuko, M. Shigematsu, T. Hashiguchi, D. Fujishima, M. Kai, N. Yoshimura, T. Yamaguchi, Y. Ichihashi, T. Yamanishi, T. Takahama, M. Taguchi, E. Maruyama, S. Okamoto. Achievement of more than $25 \%$ conversion efficiency with crystalline silicon heterojunction solar cell//IEEE Journal of Photovoltaics 4, p.1433-1435 (2014).
2. G. Rupprecht. Untersuchungen der elektrischen und lichtelektrischen Leitfähigkeit dünner Indiumoxydschichten // Z. Phys. 139 (5), p. 504517 (1954).

3. N. Balasubramanian and A. Subrahmanyam, "Electrical and Optical Properties of Reactively Evaporated Indium Tin Oxide (ITO) Films Dependence on Substrate Temperature and Tin Concentration", Journal of Physics D: Applied Physics, 22, p. 206 - 209 (1989).

4. J. C. C. Fan and J. B. Goodenough, "X-Ray Photoemission Spectroscopy Studies of Sn doped Indium Oxide Films"Journal of Applied Physics, 48(8), p. 3524 - 3531 (1977)

5. A.P. Gorban, V.P. Kostylyov, A.V. Sachenko et al. Impact of Excess Charge Carrier Concentration on Effective Surface Recombination Velocity in Silicon Photovoltaic Structures // Ukr. J. Phys. 51(6), p. 598-604 (2006)..

6. M.I. Yernaux, C. Battocchio, P. Verlinden, F. Van De Wiele. A one-dimensional model for the quantum efficiency of front-surface-field solar cells// Solar Cells. 13, p. 83-97 (1984).

7. W.P. Mulligan, D.H. Rose, M.J. Cudzinovic et al. Manufacture of Solar Cells with 21\% Efficiency // Proc. 19th European Photovoltaic Solar Energy Conf. - Paris, France, 2004. P. 387-390.

8. A.P. Gorban, V.P. Kostylyov, A.V. Sachenko, O.A. Serba, I.O. Sokolovskyi, V.V. Chernenko. Effect of Floating $p-n$ Junctions on the Efficiency of Silicon Back Side Contact Solar Cells // Ukr. J. Phys. 55(7), p.783-790 (2010).

9. V.P. Kostylyov, V.G. Litovchenko, A.V. Sachenko, T.V. Slusar, V.V. Chernenko. Features of solar cells and solar silicon wafers surface photovoltage spectral dependences in the short-wave absorption region. Proceedings 28 European Photovoltaic Solar Energy Conf. and Exhibition, Paris, France, 30 Sept. - 04 Oct., 2013.- P.1715-1718. 